\title{
Modeling and Analysis of a Glass Façade Robot
}

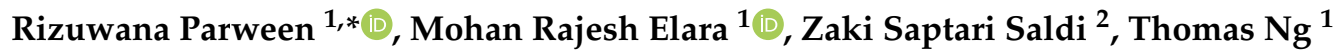 \\ and Madan Mohan Rayguru ${ }^{1}$ (D)
}

1 Engineering Product Development, Singapore University of Technology and Design, 8 Somapah Road, Singapore 487372, Singapore; rajeshelara@sutd.edu.sg (M.R.E.); thomas_ng@sutd.edu.sg (T.N.); madan_rayguru@sutd.edu.sg (M.M.R.)

2 Product Design Engineering, Universitas Prasetiya Mulya, Kavling Edutown I.1 BSD City, Tangerang 15339, Indonesia; zaki.saldi@prasetiyamulya.ac.id

* Correspondence: rizuwana_parween@sutd.edu.sg

check for updates

Citation: Parween, R.; Elara, M.R.; Saldi, Z.S.; Ng, T.; Rayguru, M.M. Modeling and Analysis of a Glass Façade Robot. Buildings 2021, 11, 225. https://doi.org/10.3390/buildings 11060225

Academic Editor: Alessandro Cannavale

Received: 18 April 2021

Accepted: 13 May 2021

Published: 24 May 2021

Publisher's Note: MDPI stays neutral with regard to jurisdictional claims in published maps and institutional affiliations.

Copyright: (c) 2021 by the authors. Licensee MDPI, Basel, Switzerland. This article is an open access article distributed under the terms and conditions of the Creative Commons Attribution (CC BY) license (https:// creativecommons.org/licenses/by/ $4.0 /)$.

\begin{abstract}
For glass façade cleaning, we developed a reconfigurable robot, Mantis-mini, with a dry cleaning mechanism and linear actuator based transitioning mechanism. It consists of three suction modules, connected by a support structure and each suction module has individual differential drive wheels with independent steering capability. This paper focuses on the detailed design of the platform, static structural analysis for and kinematic formulation. The adhesion performance of the suction module is evaluated using experiments, Computational Fluid Dynamics (CFD) simulations, and analytically formulated estimation. The suction pressure magnitude predicted from the simulations increases with suction impeller speed and agrees well with measurements and analytical calculation in terms of order of magnitude and qualitative trend. An adaptive proportional-integral (PI) controller is designed and implemented for regulating suction pressure and motion controller of the platform. The suction performance of the platform is validated through real-time experiments of the platform on the glass surface.
\end{abstract}

Keywords: glass façade cleaning robot; wheel locomotion; structural analysis; suction modeling

\section{Introduction}

High rise buildings with glass façade are springing up very commonly around the world [1]. Inspection and cleaning the glass façades is a challenging, costly, and dangerous process. Cleaning is also highly dependent on environmental conditions such as sunlight. As shown in Figure 1, the workers are tied to a rope to prevent them from falling off the side of the building. During cleaning, there is a high risk of workers hanging from the great heights and workers have fallen to death while cleaning windows at high-rise. Replacing human labour with a robotic system assisted by human operators from the ground could be an alternative for this task. This would save considerable amount of time and human effort, and also reduce the risk of fatalities. Hence, there is a need of developing "glass façade cleaning robot".

The glass façade of any building consists of several flat glass panels interconnected with frames made of metal or cement. For cleaning of the glass façade, the robot must have the ability to adhere and locomote over the glass panel. For efficient cleaning, the robot must cover the entire glass panel, which is termed as the "area coverage". Since the robot is operated from the ground, it must also cross (or transit) the metallic frame without any human intervention. The performance of the glass façade cleaning robot is measured in terms of the locomotion ability on the glass panel, transitioning across the glass frame, and maximizing the area coverage. To achieve these performance level, the first reliable solution is to incorporate a mechanism which helps the robot cross the window frame. The second solution is to implement efficient control algorithms for the enhancement of adhesion and locomotion ability. The third solution is to implement energy-efficient optimal path planning strategy to maximize the area coverage performance. 


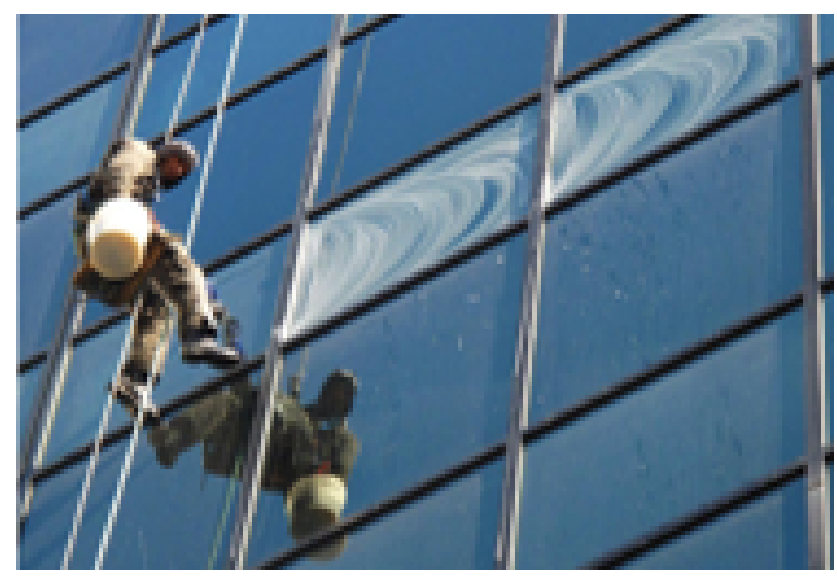

Figure 1. Workers cleaning the glass façade [2].

Due to development of automation, sensors, and Internet of Things, several research groups and industries have started developing tele-operated and autonomous robots for cleaning and inspection of vertical surfaces including cemented walls, pillars, poles and glass façade and the exterior surfaces of ship and aircraft. These robots for vertical surfaces have two key features: (i) locomotion mechanism based on wheels, chain track, multilegged, and sliding tracks and (ii) adhesion mechanism based on passive suction cups, impellers, magnetic base, magnetic wheels, adhesive pads. Over the past few years, many robotic solutions have been proposed for window cleaning and glass façade cleaningclimbing mechanisms and autonomy [3,4]. For example, a pneumatic climbing robot Sky Cleaner 3 was introduced [5]. Sky Cleaner 3 uses a water-based cleaning system connected to a wet nurse from the ground and a crane on top [6]. Kim et al., presented a novel wall-climbing robot, ROPE RIDE [7], a wall-climbing robot that navigates with the help of a crane and ropes. Nazim et al., presented a climbing robot that uses suction cups, water-based cleaning, and brushes [8]. Houxiang et al., presented a robot that uses suction cups to move through the window [9]. Dual-purpose robots have even been developed that can clean floors and windows by spraying water [10]. Hobot, Winbot, and Mamibot W-series [11-13] are the most popular robots in the market with a single module using a microfiber cloth for cleaning and spray for window cleaner liquid. Gladwell is a mopping robot for windows using differential rotation and dry cleaning mopping for locomotion [14]. Tun et al., developed a glass façade robot with passive suction cups and auto-locking lead screws [15]. Bioinspired climbing mechanisms are also considered for vertical façade cleaning [16]. Anwari et al., discussed about the use of a pneumatic glass façade cleaning robot with an impeller as a suction tool to hold robot against the glass façade [17]. All these robots are fixed form robots, whose shape and adhesion area remain fixed throughout the navigation process. These robots can only clean the glass panels and are not capable to cross the window frames.

In order to overcome current limitations of vertical robots and adapt to glass panels along with crossing the window frames, a number of research groups have applied the concept of reconfiguration to develop glass façade robots. Our team has also applied "theory of reconfigurability" while designing mechanism for floor cleaning [18-22], drain mapping [23], pavement sweeping [24] and vertical pillar inspection [23]. These platforms incorporate 'robust switch-based controller, adaptive controller', and the experimental data results give good results as compared to simple PID controller. Hence, the same reconfigurability concept can be applied to develop a glass façade cleaning robot. For example, Nansai et al., proposed a reconfigurable window cleaning robot using suction cups and water-based cleaning [25]. Vega et al., fabricated a glass wall-climbing robot, Mantis, that can shift from one window panel to another [26] and has a multisensory system for positioning and is able to detect cracked glass [27]. However, these reconfigurable robots are only designed for flat glass surfaces and do not have the capability to navigate on curved glass surfaces. These robots use PD controllers for navigation and adhesion. 
These robots lack the performance of a detailed controller design. Hence, a closer look needs to be taken to enhance the robot performance so that it can adapt and clean flat glass surface. In order to achieve this, studying the controller design, and the enhancement of the robot locomotion and adhesion performance of the robot is required.

In this paper, we present the design and developmental aspects of the miniaturized Mantis platform named Mantis-mini. This is a reconfigurable and modular glass façade robot that has the ability to adapt and clean flat glass surfaces and cross the window panel. The contribution of this work lies in the analysis on modeling of the suction chamber along with an adaptive controller suitable for the platform. The paper discusses the modelling, analysis, and controller design. The remainder of the paper is organised as follows. Section 2 outlines the system architecture. Sections $3-5$ outline the structural analysis, fluid simulation of the suction chamber and platform kinematics, respectively. Section 6 presents the experimental studies of the platform. Section 7 outlines the conclusion.

\section{System Architecture}

In the first phase of the project, the platform is fabricated and the basic functionalities including the suction and locomotion of the platform on various surfaces including glass, wood, and cement are verified. The platform architecture is explained as follows. Figure 2 shows the CAD model of the platform. The platform consists of three independent modules. Each module has a holder and all three holders are interconnected by support frames. Each module has an independent locomotion unit which follows differential drive principle and the locomotion unit has the ability to steer about its own axis wheel. Each module is connected to three support frames by a holder and linear actuator. The frame gives mechanical stability to the whole system and also connects all three modules and holders. The holder is placed in the upper part of the suction module that connects the frame and the Module. The holder also provides in-plane rotation of the modules with the help of ball bearings. Figure 3 shows the detailed view of single suction module. Each modules has a suction chamber containing an impeller that generates suction force based on differential pressure. The impeller has a nominal voltage rating of $24 \mathrm{~V}$ and is run by a DC motor with voltage rating of 12-24 V. The pressure inside the suction chamber helps to adhere the platform to the glass panel.

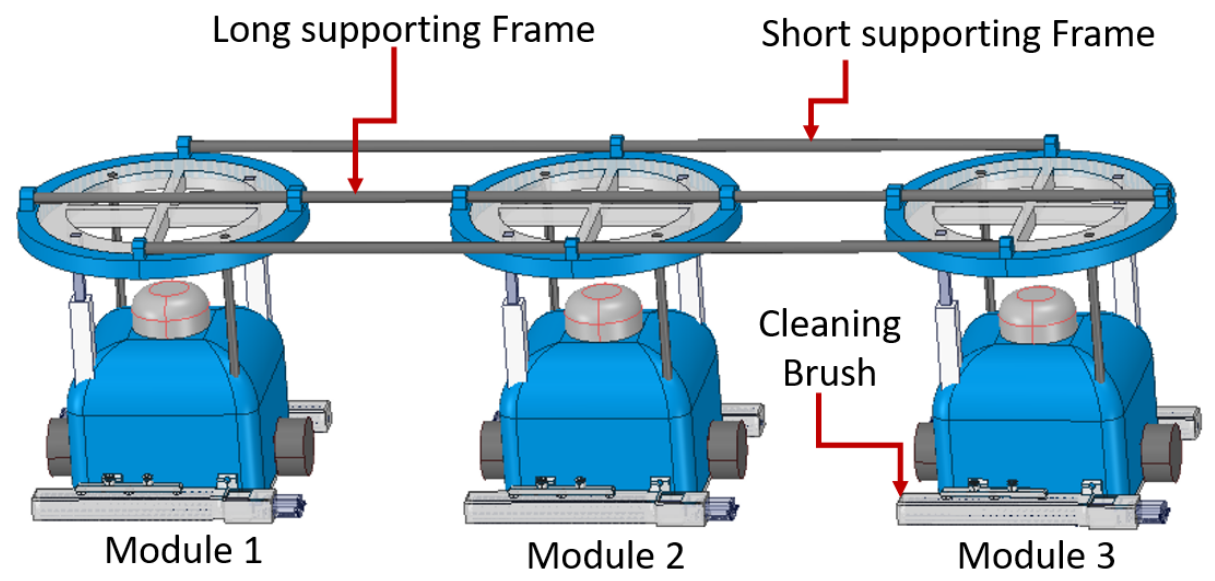

Figure 2. CAD model of the entire Mantis-mini.

The lifting mechanism needs to be designed in such a way that the lifting movement of the module while transitioning should not affect the long and short supporting frame. In this design, the lifting mechanism consists of two linear actuator placed diagonally, by which the suction module can be lifted up towards the main support frame. The lifting mechanism consists of an L16-S miniature linear actuator which has an extension length of $100 \mathrm{~mm}$ with 35:1 gear ratio with a power supply of $12 \mathrm{~V}$. Each module holds two linear actuators which help in giving the clearance for the module during the transition process. 
The linear actuator offers a efficient mechanism. To ensure the stability of each module being lifted and lowered onto the glass panel, they are also fitted with carbon fibre rods, which helps to ensure a smooth and straight trajectory for the module. Carbon fibre is very strong and lightweight, which makes it ideal to be a guide for the linear actuator.

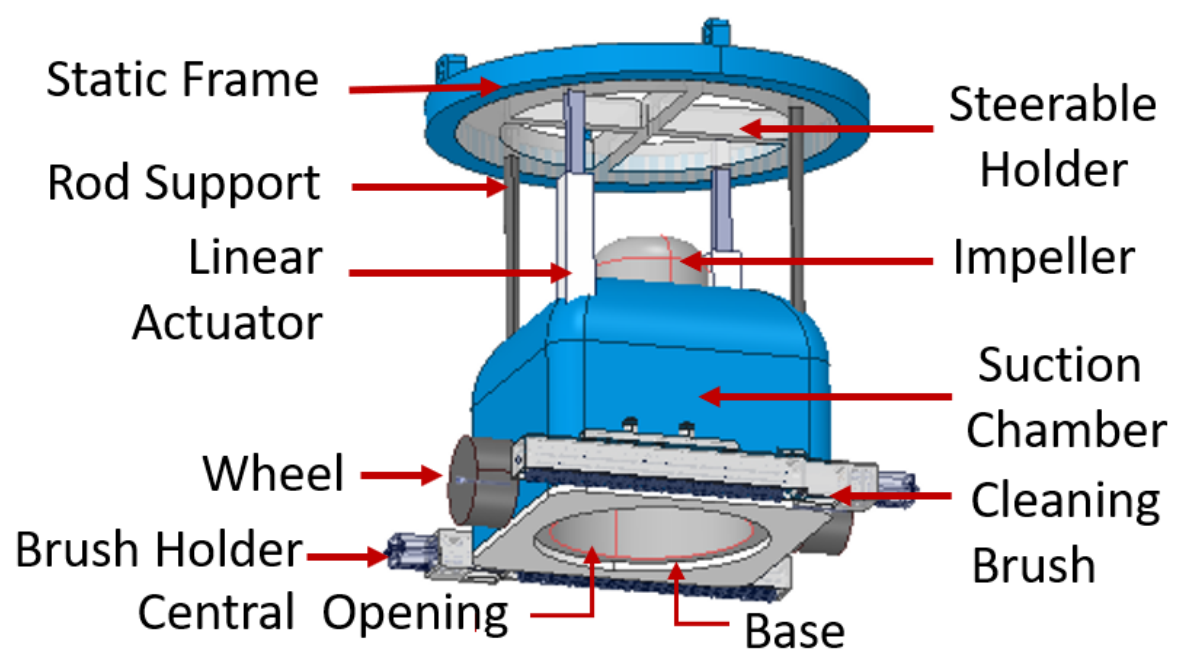

Figure 3. CAD model of the suction module of the entire Mantis-mini.

The objective of the Mantis-mini platform is to clean a glass window panel and transit the next panel without human intervention. For this platform to transit to the next panel successfully, any two modules must hold the system on the surface of the façade and must be sufficient to drive the system. Hence, the contact area of the platform on the façade changes during transitioning. After transitioning, the modules align themselves on the façade and the robot resumes cleaning of the second window. Figure 4 shows the different configurations of the Mantis-mini platform during panel transitioning. There are two phases during the transition. Firstly, two modules are in contact with the first window panel, driving the entire platform across to the next window panel. Secondly, the linear actuator will lower the first module onto the next panel and this is done until all 3 modules have crossed over to the second panel.

Each module has a differential drive which enables it to rotate on its own. The differential wheel drives and a ball bearing attached to the platform aids in both transition and locomotion. The modules would have to rotate itself individually so that the entire platform would be able to traverse in the $\mathrm{x}$ and $\mathrm{y}$-direction along the glass panel. The frame holding the entire platform would remain horizontal during all scenarios which means the modules are able to rotate on their own without rotating the frame. This is possible with the help of ball bearings attached at the holder, using the differential wheels to rotate the module.

Figure 5 shows the electronics block diagram of Mantis-mini platform. The platform is powered by a $24 \mathrm{~V} \mathrm{DC}$ power supply that drives the impellers of each modules and the motor drivers of the wheels. They are controlled using an Arduino Mega to send out commands for each motor driver and impellers. A $2 \times 7$ A Roboclaw motor driver and a voltage relay compatible with $3.3 \mathrm{~V}$ and $5 \mathrm{~V}$ appliances are used. The locomotion unit consists of two Solarbotics RW2i rubber robot wheel with thick silicone rubber tire for maximum traction. This wheel features an internal set screw hidden underneath the tire. This saves valuable space when designing robots with size constraints. The wheels are driven by two Pololu micrometal gear motors. These gear motors are a miniature high-powered, $12 \mathrm{~V}$ brushed DC motor with long-life carbon brushes and a 248.98:1 metal gearbox. It has a cross section of $10 \mathrm{~mm} \times 12 \mathrm{~mm}$, and the D-shaped gearbox output shaft is $9 \mathrm{~mm}$ long and $3 \mathrm{~mm}$ in diameter. 

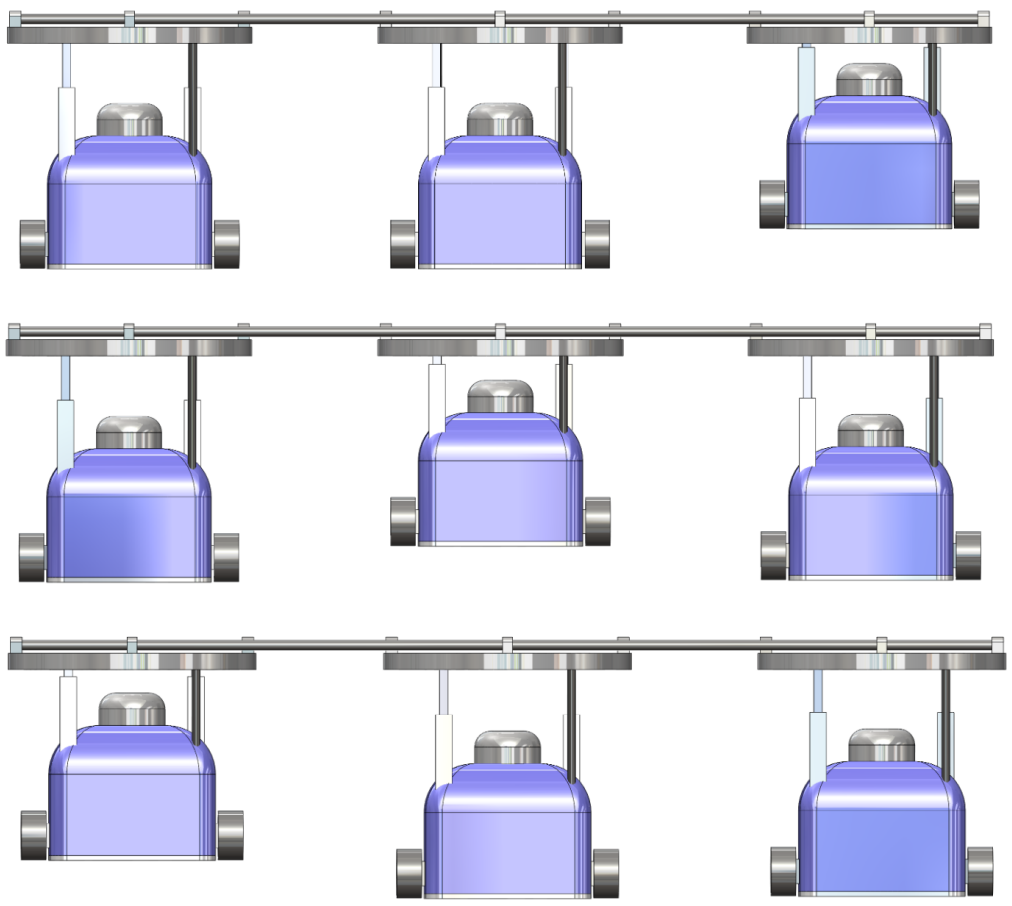

Figure 4. Transitioning steps during crossing the window frame.

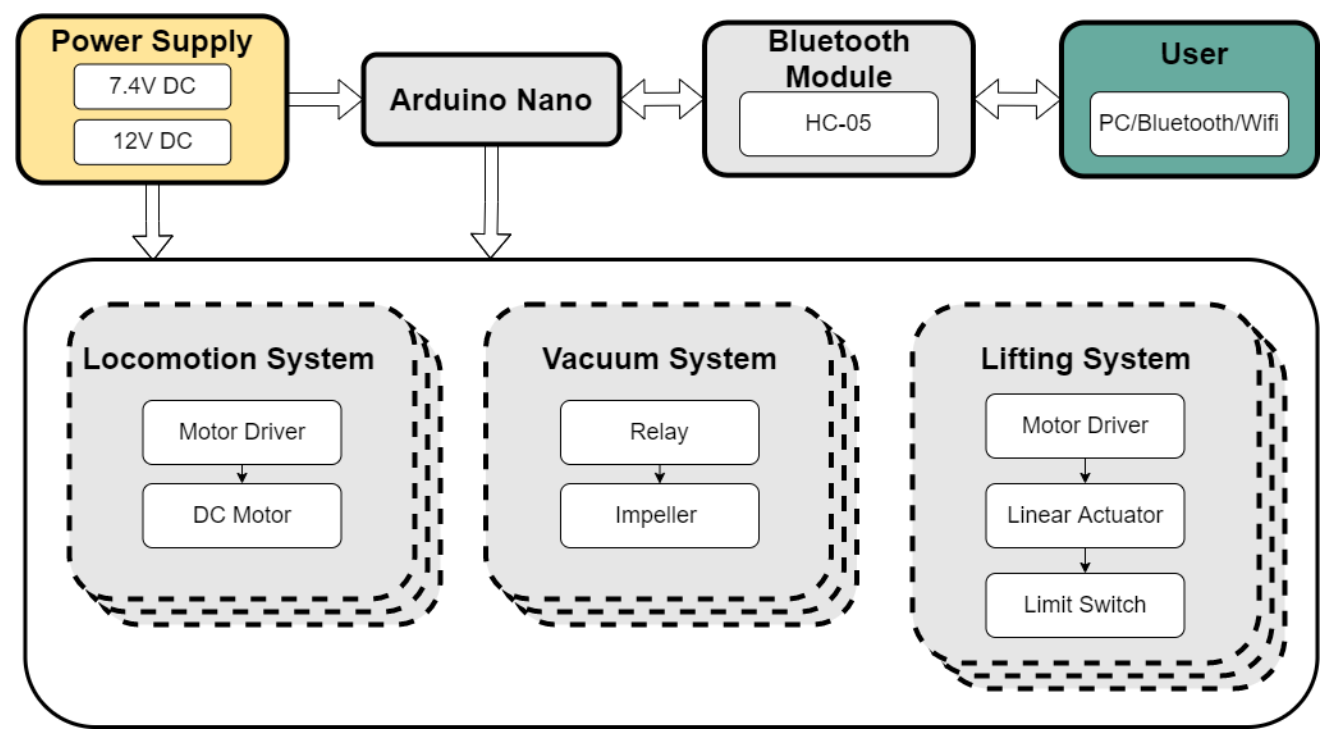

Figure 5. Electronics block diagram of Mantis-mini.

Figure 6 shows the fabricated prototype of the Mantis-mini. The suction module and the holder is made of acrylic and the support frames are made of carbon fiber. These help to reduce the overall weight of the platform. The dimensions of each suction module is $100 \mathrm{~mm} \mathrm{~L} \times 100 \mathrm{~mm} \mathrm{~W} \times 95 \mathrm{~mm} \mathrm{H}$ and the total dimensions of the entire platform is $600 \mathrm{~mm} \mathrm{~L} \times 150 \mathrm{~mm} \mathrm{~W} \times 153 \mathrm{~mm} \mathrm{H}$. The total weight of the platform is $8 \mathrm{~kg}$. The platform is remotely navigated using a mobile application to include all approaches needed to accomplish a successful glass façade cleaning operation. 


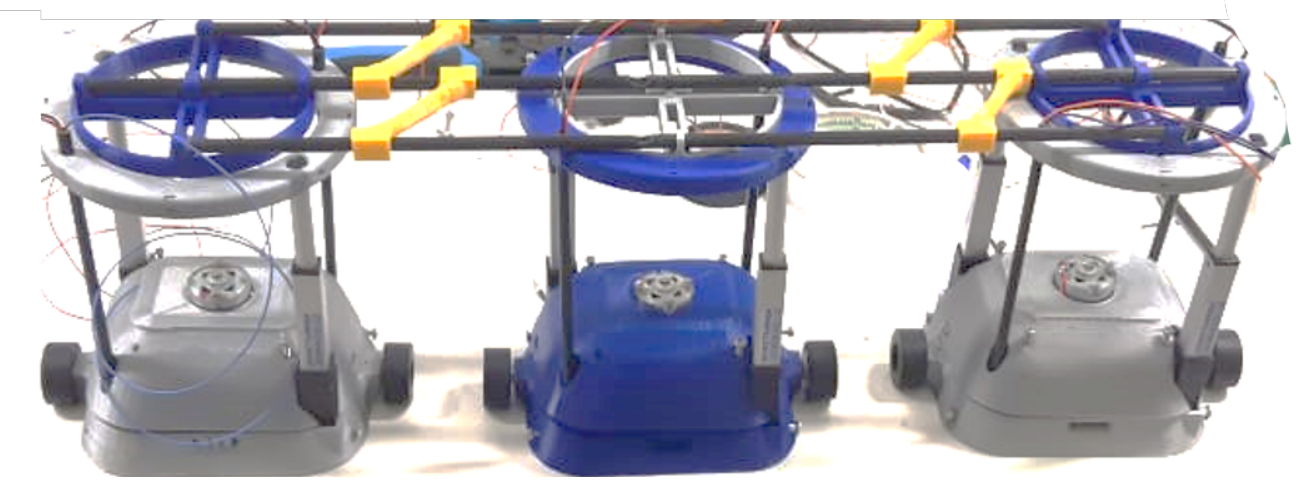

Figure 6. Prototype of Mantis-mini.

\section{Structural Analysis}

Natural frequency is a critical parameter of any oscillating system. When a dynamical system is driven by an external force at any of its natural frequency of the system, then the system is under resonance and undergoes structural failure. Even though the structural platform of the robot is made of rigid material, cleaning pay load and friction on the surface may add additional load to the motor and cause failure due to resonance. Therefore, during locomotion and transitioning of the platform, the actuation speed of the wheel motor and impeller motor must be set less than the first natural frequency of the system to avoid resonance. This robot platform has two different mode of operation, i.e., static and locomotion modes. The static condition refers to a case when the platform is adhered to wall with suction force. During static and locomotion mode, the main contact point of the robot on walls are the wheel peripheral surface and the adhesion area of the suction cup. This section presents the modal and static analysis of the platform.

\subsection{Frequency Analysis}

A simplified CAD model of the platform, shown in Figure 2 is considered for analysis. For finite element analysis in ANSYS workbench version (2020 R1), the model is discretized with solid 187 elements. A modal analysis is carried out to obtain the natural frequencies and mode shapes of the platform during static condition with cleaning system. At these contact points, a fixity condition of $u_{x}=u_{y}=u_{z}=0$ is applied. Figure 7 shows the mode shapes and the corresponding natural frequency of the platform. The first natural frequency represents the in-plane torsional oscillation of the platform about the wheels. The second mode is the in-plane bending of both side blocks about its middle block. The third mode of oscillation is the out-of-plane torsional oscillation of the entire platform. Hence, in these modes, the supporting frames and the static frame, and steerable frames are the critical region. The lowest natural frequency of the platform with the cleaning module is found to be $92 \mathrm{~Hz}$. For structural safety, the impeller and the cleaning motor must be operated at a frequency lower than the first natural frequency.

\subsection{Static Analysis}

A static structural analysis is carried out in Workbench environment to study the gravitational $(1 \mathrm{~g})$ effect on the platform. A payload of $2.5 \mathrm{~kg}$ is applied as a uniformly distributed load on the base plate of the platform. Fixity boundary condition is applied at the ground-wheel contact point of the platform. The factor of safety and the deformed components of the platform are shown in Figures 8 and 9. The maximum deformation is $24 \mathrm{~mm}$, which occurs near the edge of the central opening of each module. This higher value is due to the stress concentration at the sharp edge of the central opening. The concentrated stress can be reduced by using filleting and providing extra material to the base. However, in other parts of the platform, the deformation is less than $5 \mathrm{~mm}$. This deformation is approximately lesser than equal to the one-tenth of the critical dimension of the platform. Figure 10 presents the distribution of the principal stress in the platform. Maximum stress of 
magnitude $40 \mathrm{MPa}$, occurs near the wheel support which is due to stress concentration factor. However, the resulting stress on the platform is about $0.4 \mathrm{MPa}$, lower than the tensile stress of PLA (40 MPa). Therefore, the current platform design is considered to be safe.

(a)
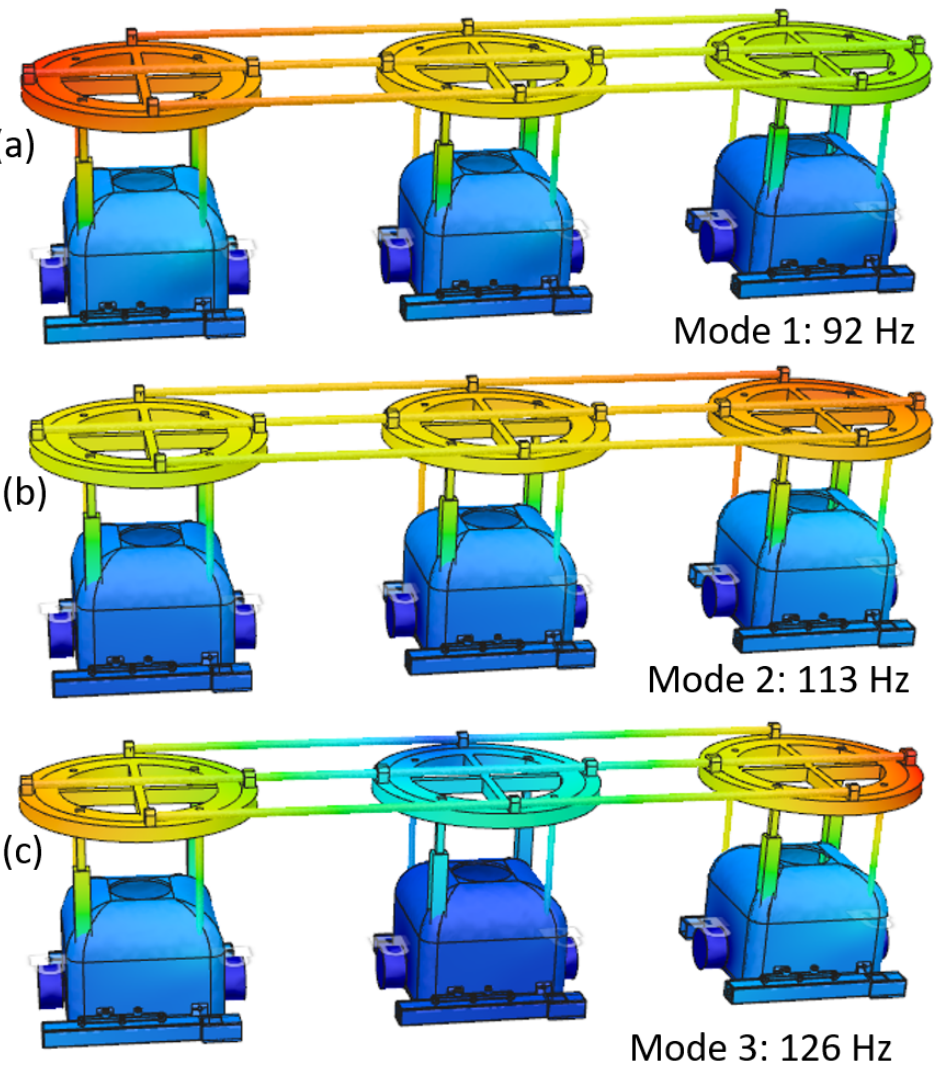

Figure 7. (a) First, (b) second, and (c) third mode shape of the platform.

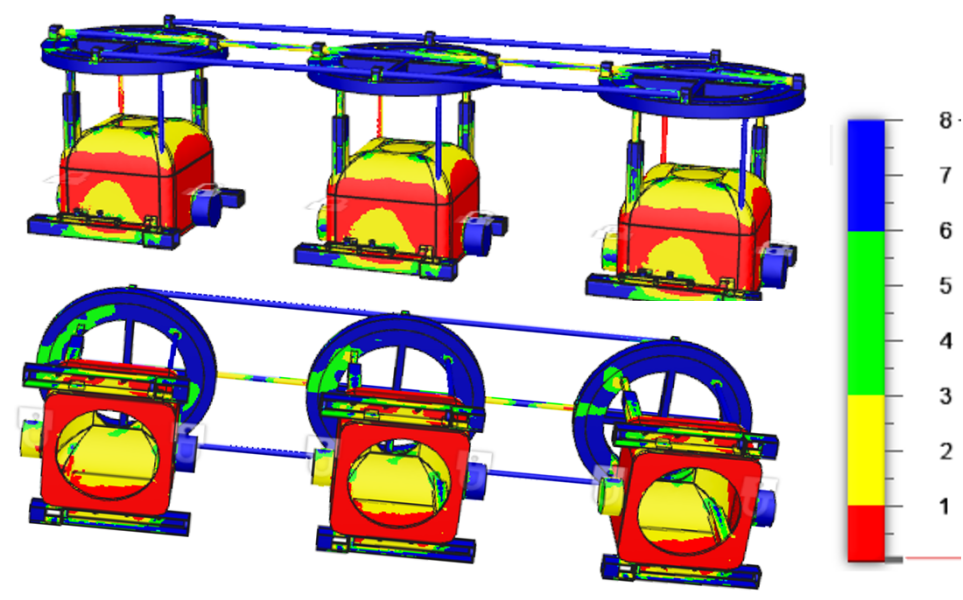

Figure 8. Factor of safety of the design. 


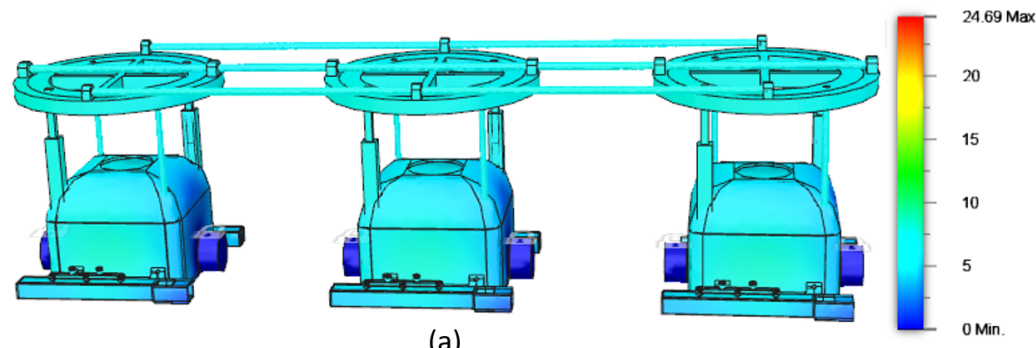

(a)

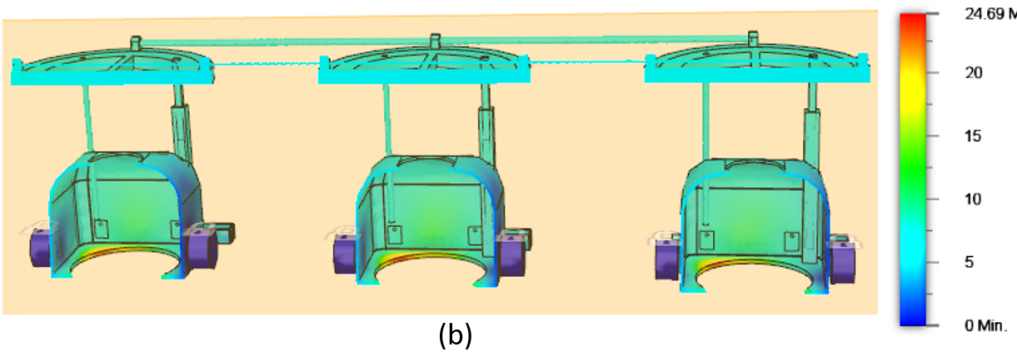

Figure 9. Deformation (mm) of the Mantis-mini platform (a) full view and (b) sectional view.

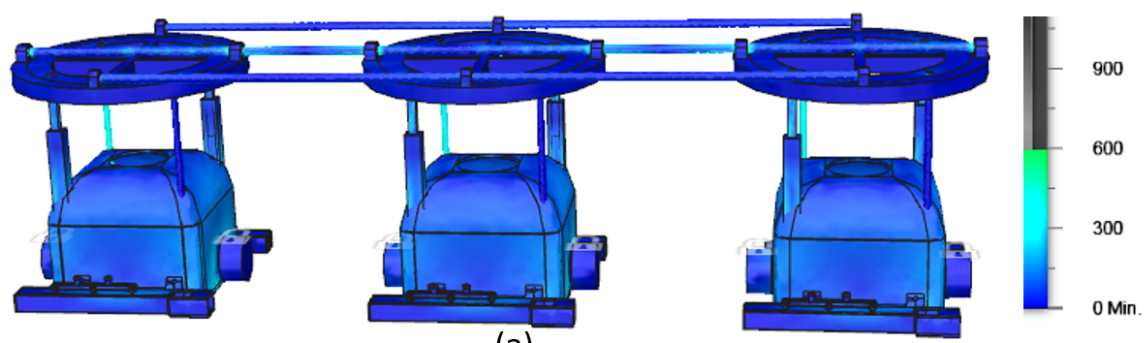

(a)

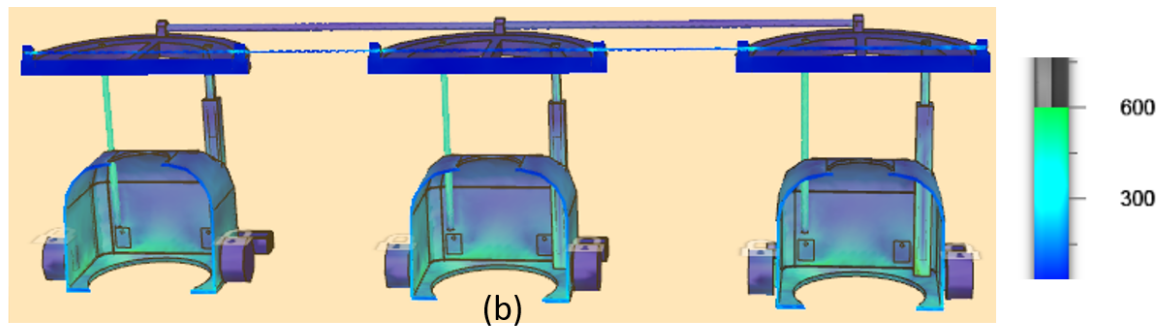

Figure 10. Stress distribution (MPa) in the Mantis-mini platform (a) full view and (b) sectional view.

\section{Modeling and Analysis of Suction}

\subsection{Analytical Formulation}

The robot capability of climbing a vertical wall is allowed by an impeller mounted just above the suction chamber, as shown in Figure 3. Due to pressure difference generated by the impeller, air flows from the opening at the base and creates suction that keeps the robot attached to the wall. The free body diagram of the robot vertically oriented to the wall is given in Figure 11a, where the following forces apply: weight $\left(F_{w}=m g\right)$, friction $\left(F_{f}\right)$, contact $\left(F_{c}\right)$, and suction $\left(F_{s}\right)$. Figure $11 \mathrm{~b}$ shows the free body diagram of the suction module. Using static equilibrium principle, the required suction pressure to prevent the robot from slipping can be derived as follow:

$$
\begin{gathered}
F_{f}=\mu \cdot F_{c}=\mu \cdot F_{s}=\mu \cdot A_{c} \cdot p_{s} \geq m \cdot g \\
p_{s} \geq \frac{m \cdot g}{\mu \cdot A_{c}}
\end{gathered}
$$



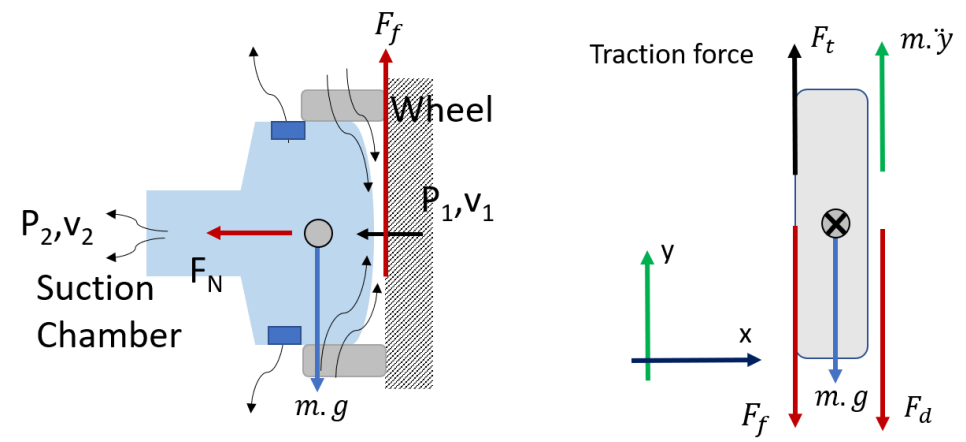

$\mathrm{F}_{\mathrm{f}}=$ Vertical Frictional force acting parallel to wall

$F_{N}=$ Reaction Support force on the suction chamber

Figure 11. (a) Free body diagram of each suction module, (b) Free body diagram of the platform during locomotion.

The suction pressure represents the pressure difference between two parallel planes, i.e., the chamber plane and the impeller plane. Pressure difference between two planes can be estimated using Bernoulli principle of steady incompressible flow, i.e.,

$$
p_{1}+0.5 \cdot \rho \cdot v_{1}^{2}+\rho \cdot g \cdot h_{1}=p_{2}+0.5 \cdot \rho \cdot v_{2}^{2}+\rho \cdot g \cdot h_{2}
$$

where subscripts 1 and 2 designate the suction chamber area and the impeller channel area, respectively, as illustrated in Figure 11. By neglecting pressure head $\left(h_{1}=h_{2}\right)$, the suction pressure can be expressed as

$$
p_{s}=p_{1}-p_{2}=0.5 \cdot \rho \cdot\left(v_{2}^{2}-v_{1}^{2}\right)
$$

The flow velocity in the impeller channel can be approximated as

$$
v_{2}=r_{2} \cdot \omega
$$

Due to mass conservation, the flow rate at Sections 1 and 2 is constant, i.e.,

$$
v_{1} \cdot A_{1}=v_{2} \cdot A_{2}
$$

Combining Equations (4)-(6) results in the following expression of suction pressure as function of the impeller rotational speed

$$
p_{s}=0.5 \cdot \rho \cdot r_{2}^{2} \cdot \omega^{2} \cdot\left[1-\left(\frac{A_{2}}{A_{1}}\right)^{2}\right]
$$

It must be underlined though that Equation (7) may not be fully applicable to predict accurately the suction pressure, as Bernoulli principle is only valid for flows where viscous effects are negligibly small compared to inertia and pressure effects. Such case is unlikely with the flow in the suction chamber, especially at high impeller speed where the flow is turbulent and rotational. However, it can be expected that Equation (7) can give a fair estimate of the order of magnitude of suction pressure and how it varies with impeller speed.

\subsection{CFD Simulation}

In order to study the effect of impeller rotational speed on the suction performance by taking into account the viscous effects, one can rely on CFD simulations. In the present work, the simulations of the airflow through the section chamber of the experimental unit 
of Mantis-mini were carried out using Solidworks Flow Simulation package, in which the conservation of mass and momentum of an viscous incompressible airflow is numerically solved.

As the main interest is in the overall suction pressure rather than in the swirling flow details caused by the impeller, the simulations can be simplified by physically excluding the impeller and accounting for its effect by setting volumetric flow rate based on the impeller speed at the top outflow boundary. Atmospheric pressure is imposed at the opening at the base and two lateral openings, while no-slip condition is imposed at the suction chamber walls.

The simulations are performed by varying the impeller rotational speeds $(4340,8760$, $12,950,17,000$, and 20,530 rpm) and the base openings ( $3 \mathrm{~cm}$ and $6 \mathrm{~cm}$ diameter). The Reynolds number is estimated to be in the range of $1.5 \times 10^{4}-7.0 \times 10^{4}$, implying turbulent flow regime. To account for turbulence, the $k-\epsilon$ model based on Reynolds-Averaged Navier Stokes (RANS) approach is used, by which the transport equations for the turbulent kinetic energy $(k)$ and its dissipation rate $(\epsilon)$ are solved.

The 3D computational domain is discretized into four levels of mesh resolution in order to evaluate the numerical solution sensitivity, i.e., M1-M4, corresponding with $170,000,490,000,990,000$, and 1,490,000 cells, respectively. The suction pressure values obtained from the simulations with base opening of $6 \mathrm{~cm}$ and impeller speed of 20,530 rpm using different mesh resolutions are shown in Figure 12. It can be observed that increasing the number of cells from M3 to M4 hardly changed the suction pressure, indicating mesh-independent solution at M3. Therefore, the simulations will be evaluated using M3 (990,000 computational cells).

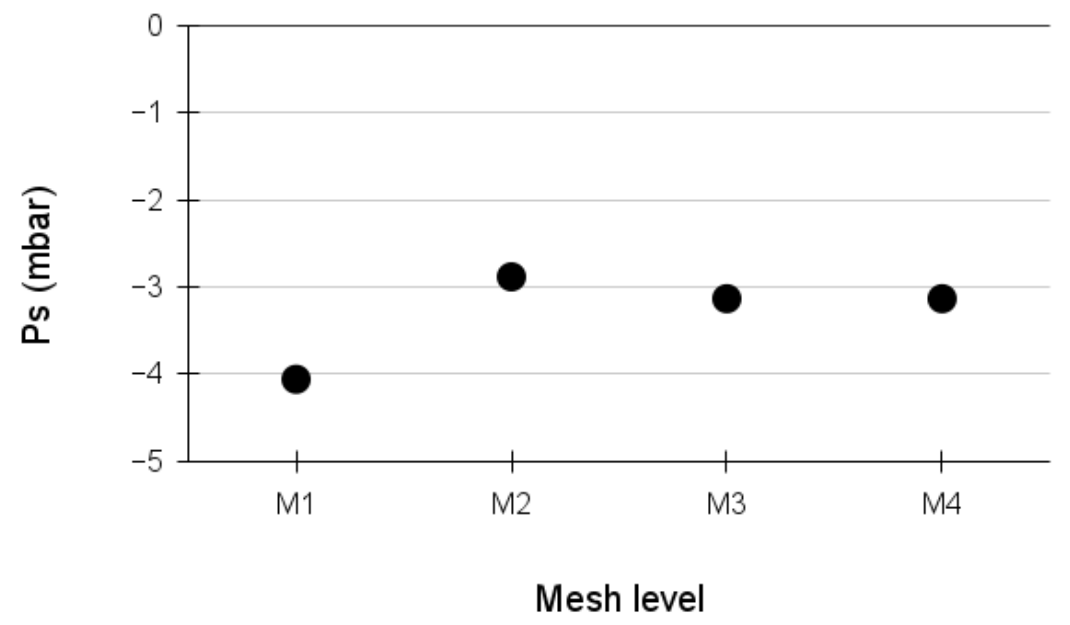

Figure 12. Suction pressure sensitivity to mesh resolution (20,530 rpm, $6 \mathrm{~cm}$ chamber base opening).

\subsection{Simulation Results}

From CFD simulations, the steady-state pressure and flow velocity fields in the suction chamber can be obtained, as shown in Figure 13 for the cross section of the chamber for the highest impeller speed $(20,530 \mathrm{rpm})$ and both base openings. The pressure fields in the suction chamber with $3 \mathrm{~cm}$ opening is more uniform in the vertical (axial) direction than in the case with $6 \mathrm{~cm}$ opening. This is attributed to a more uniform velocity field in the axial direction for the $3 \mathrm{~cm}$ case. In the $6 \mathrm{~cm}$ case, velocity gradients in the axial direction is more pronounced. Accordingly, the pressure drop from the base opening towards the top outlet boundary in the $6 \mathrm{~cm}$ opening configuration is higher.

The suction pressure for varying impeller speed can also be calculated from the simulations. The relation between suction pressure and impeller speed obtained from simulations is compared with experiment and theoretical calculation results, and is explained in Section 6. 
(a)

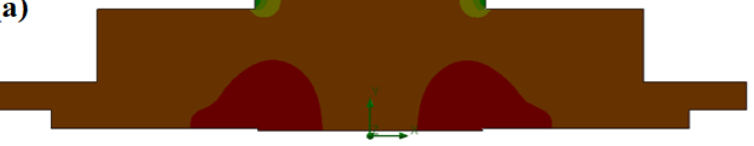

(b)

(b)

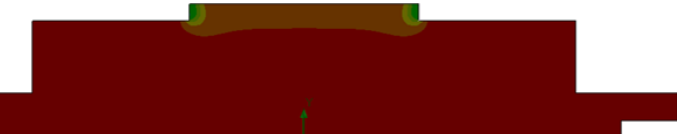

c)

(c)

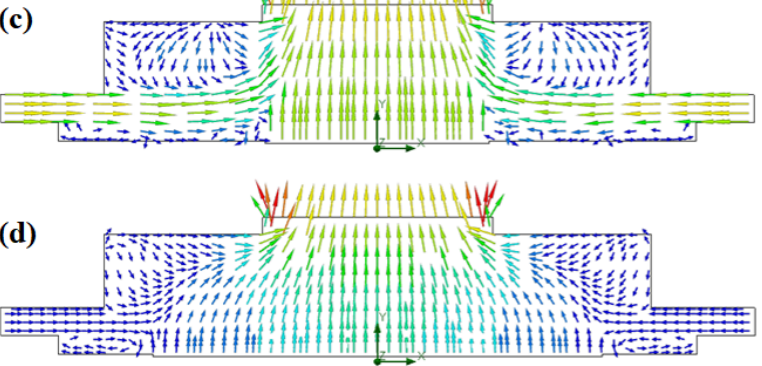

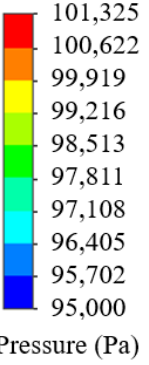

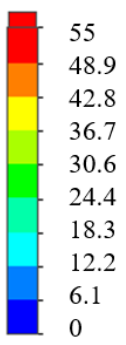

Velocity $(\mathrm{m} / \mathrm{s})$

Figure 13. Cross-sectional views of steady-state fields at 20,530 rpm: (a) Pressure, $3 \mathrm{~cm}$ opening; (b) Pressure, $6 \mathrm{~cm}$ opening; (c) Velocity vectors, $3 \mathrm{~cm}$ opening; (d) Velocity vectors, $6 \mathrm{~cm}$ opening.

\section{Platform's Kinematics}

As mentioned before, the Mantis-mini platform consists of three identical modules with an independent differential-drive for each. There are therefore six wheels in total in the platform. For the navigation on the glass panel, we use the simplified kinematic equation based on on differential drive principle. A set of three wheels on the left side $\left(w_{1}, w_{2}, w_{3}\right)$ wheel and three wheels on the right side $\left(w_{4}, w_{5}, w_{6}\right)$ wheel are represented by a virtual wheel $w_{L}$ and $w_{R}$ respectively. $\beta_{j}$ is the angular orientation of each module $(j=1,2,3)$ shown Figure 14 . The robot is able to change its orientation by varying the relative rate of rotation of these virtual wheels. Figure 14 shows the schematic diagram of the wheel layout which is used for kinematic analysis. Let $L_{i}$ denotes the distance between robot inertial system and wheels' point of contact. $\dot{\phi}_{i}$ is the angular velocity of $i$ th wheel. $v_{R}$ and $v_{L}$ are the linear velocity of the virtual right and left wheel, respectively. $v_{p}, \omega_{p}$ are the translation and angular velocity of the robot platform about its center of mass respectively. The following equations govern the kinematics relations.

$$
\begin{gathered}
v_{L}=r \frac{\dot{\phi}_{1}+\dot{\phi}_{2}+\dot{\phi}_{3}}{3} \\
v_{R}=r \frac{\dot{\phi}_{4}+\dot{\phi}_{5}+\dot{\phi}_{6}}{3} \\
v_{p}=\frac{v_{L}+v_{R}}{2} \\
\omega_{p}=\frac{\left(\dot{\phi}_{1} \cdot C_{1}+\dot{\phi}_{2} \cdot C_{1}+\dot{\phi}_{3} \cdot C_{2}\right)-\left(\dot{\phi}_{4} \cdot C_{2}+\dot{\phi}_{5} \cdot C_{3}+\dot{\phi}_{6} \cdot C_{3}\right)}{\sum_{1}^{6} L_{i}}
\end{gathered}
$$

where $C_{i}$ is cos of the steering angle of the wheel in the module 1,2,3 respectively. The position of the center of the mass of the platform is $\left(x_{c}, y_{c}\right)$. The distance between the wheels of each module and the centre of each module is denoted as $l$.

$$
\begin{aligned}
& x_{c}=\int_{o}^{t} v_{p} \cdot \cos \alpha d t \\
& y_{c}=\int_{o}^{t} v_{p} \cdot \sin \alpha d t
\end{aligned}
$$




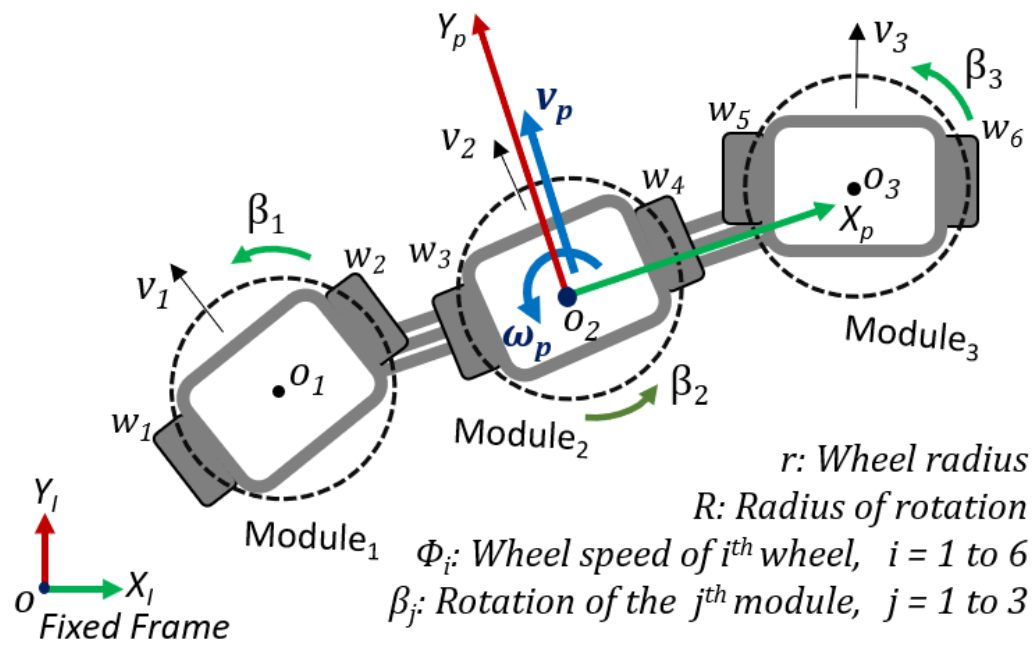

Figure 14. Schematic diagram of the Mantis-mini presenting the wheel layout.

\section{Results and Discussion}

\subsection{Variation of Adhesion Performance with Speed}

Figure 15 shows the experimental set up that was used to measure the pressure and the force exerted by the impeller over the glass substrate. The independent variables are the substrate, the size of the opening at the bottom of the chamber, and the speed of the motor. This is to analyze how Mantis-mini would perform over different surfaces, and find out how different opening sizes affect the pressure within the chamber, as well as to see the relationship of the average force and pressure over varied speed. As for the opening size at the bottom of the chamber, experiments involving $3 \mathrm{~mm}$ and $6 \mathrm{~mm}$ were used. In addition, the force sensors are used to measure the contact force of the suction chamber on the glass surface.

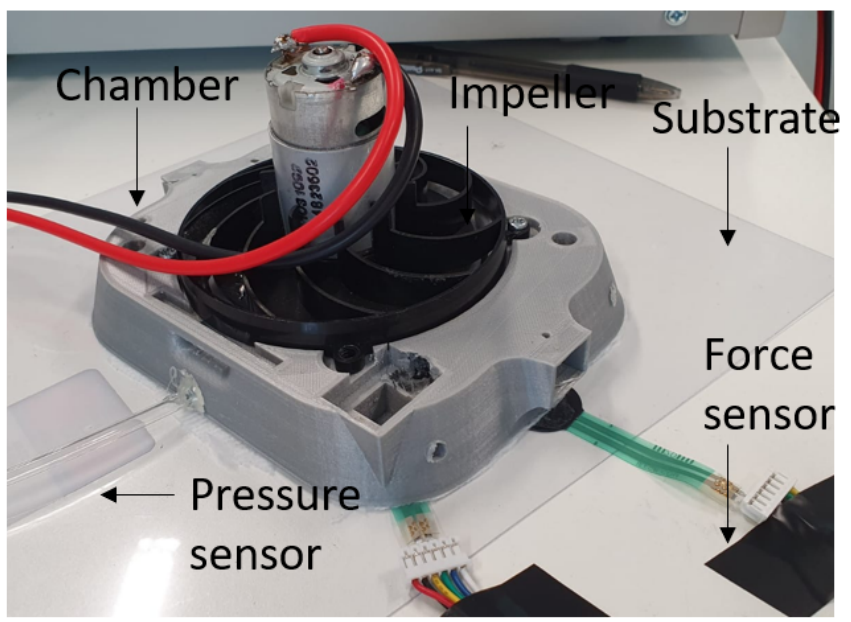

Figure 15. Experimental set up for pressure and contact force measurement.

Figures 16 and 17 depict how the pressure within the chamber varies with the motor speed with $3 \mathrm{~cm}$ and $6 \mathrm{~cm}$ opening, respectively. The pressure sensor inside the chamber was placed near the center, close to the top outlet boundary. The qualitative trend of the prediction using simulation, experiment, and analytical formula agree well with each other, i.e., the suction pressure magnitude increases with impeller speed and the opening size. Nevertheless, there is an obvious difference among the three, which can be explained as follow. 


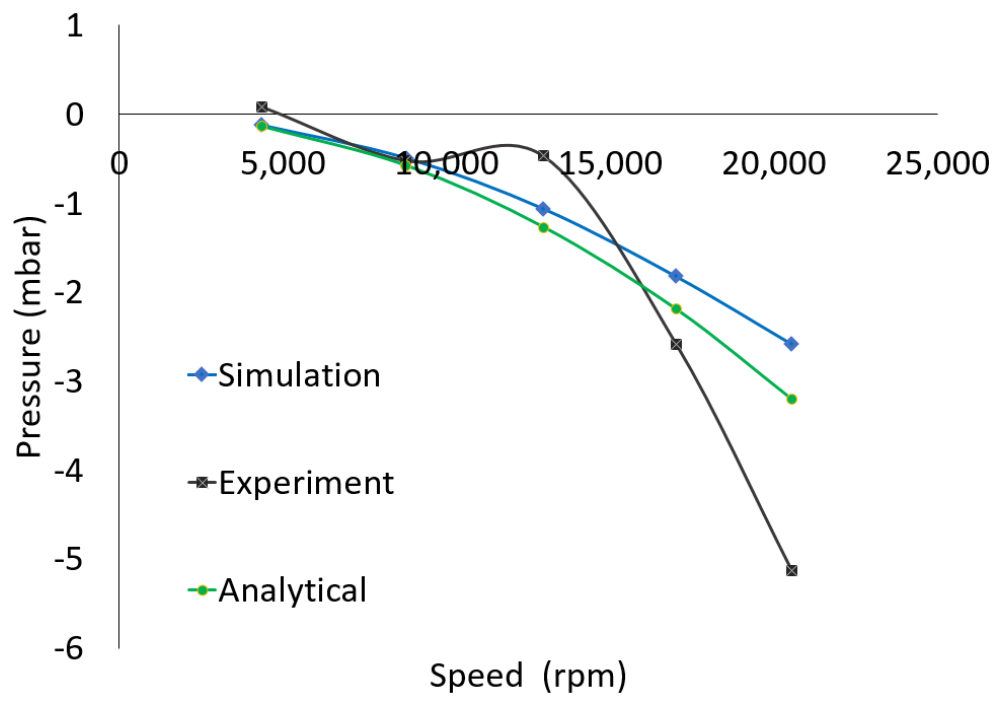

Figure 16. Pressure variation for $3 \mathrm{~cm}$ opening of the suction chamber.

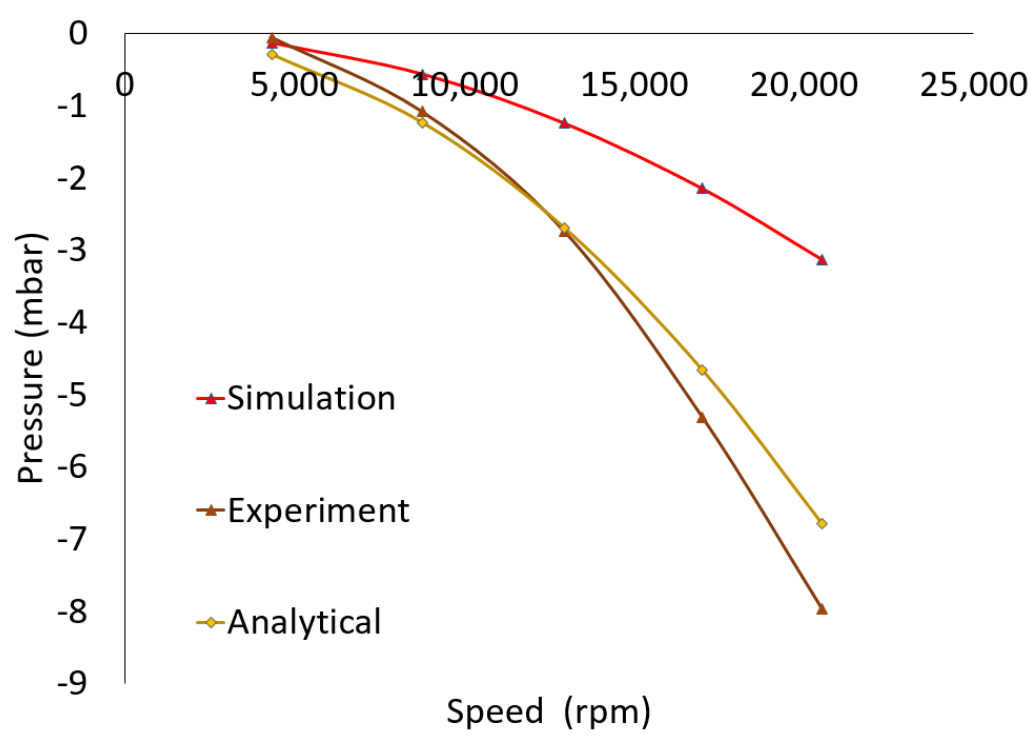

Figure 17. Pressure variation for $6 \mathrm{~cm}$ opening of the suction chamber.

The suction pressure from the simulation is obtained by taking the difference between the surface average pressure at the top outlet and bottom inlet at steady state, whereas the experimental values are based on average values of point measurement. This difference resulted in the discrepancy in the suction pressure magnitude between simulation and experiment. The discrepancy is also larger at higher impeller speed and for the $6 \mathrm{~cm}$ opening. Prediction using the analytical formula (Equation (7)) closely matches the simulation for the $3 \mathrm{~cm}$ opening and the experiment for the $6 \mathrm{~cm}$ opening. It has to be noted that the formula is not valid for turbulent flow, which is more dominant with higher impeller speed. This can explain the larger difference between analytical prediction and simulation/experiment at impeller speeds over 15,000 rpm.

Figure 18 depicts how the contact force exerted by the chamber varies with motor speed. Although there are 2 force sensitive resistors (FSR) being placed beneath the chamber as shown in Figure 15, where one FSR is placed at the edge while the other is closer to the center, it was observed that the FSR at the center does not register any value, or that there is minimal force in the center. Hence the force values that are being used in the graph are from the edge of the chamber. 


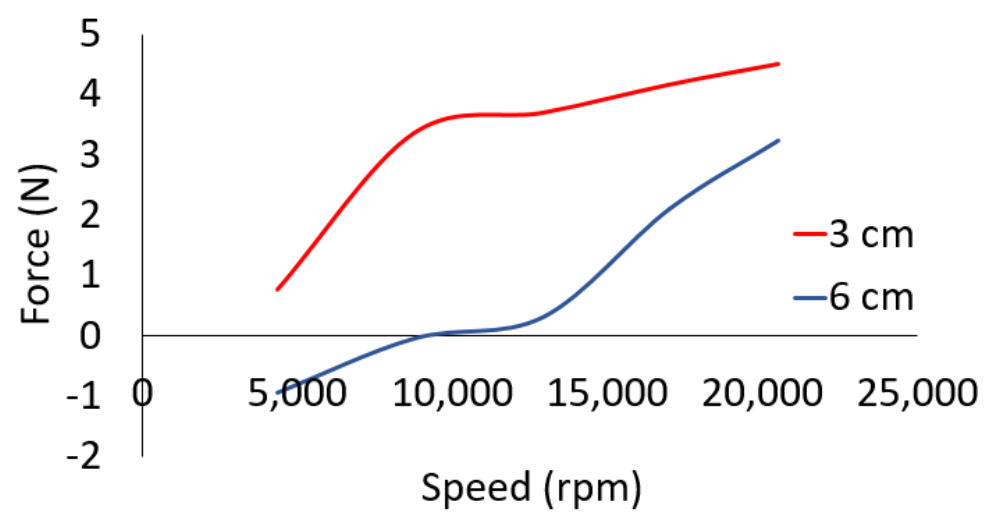

Figure 18. Contact force variation for $3 \mathrm{~cm}$ and $6 \mathrm{~cm}$ opening of the suction chamber.

\subsection{Controller Selection}

This platform incorporates a low level actuator control for ensuring proper adhesion and locomotion along a particular type of vertical plane. It must be noted that the impeller is responsible for providing negative pressure so that the platform does not lose contact with the vertical surface, whereas the motion is supported by a standard DC motor. Hence, the controller objectives are to regulate a required output pressure for the impeller and to regulate a desired speed for the DC motor. As the actuators responsible for these tasks are not the same, the controller design can be decoupled. The proposed controller architecture is presented in Figure 19.

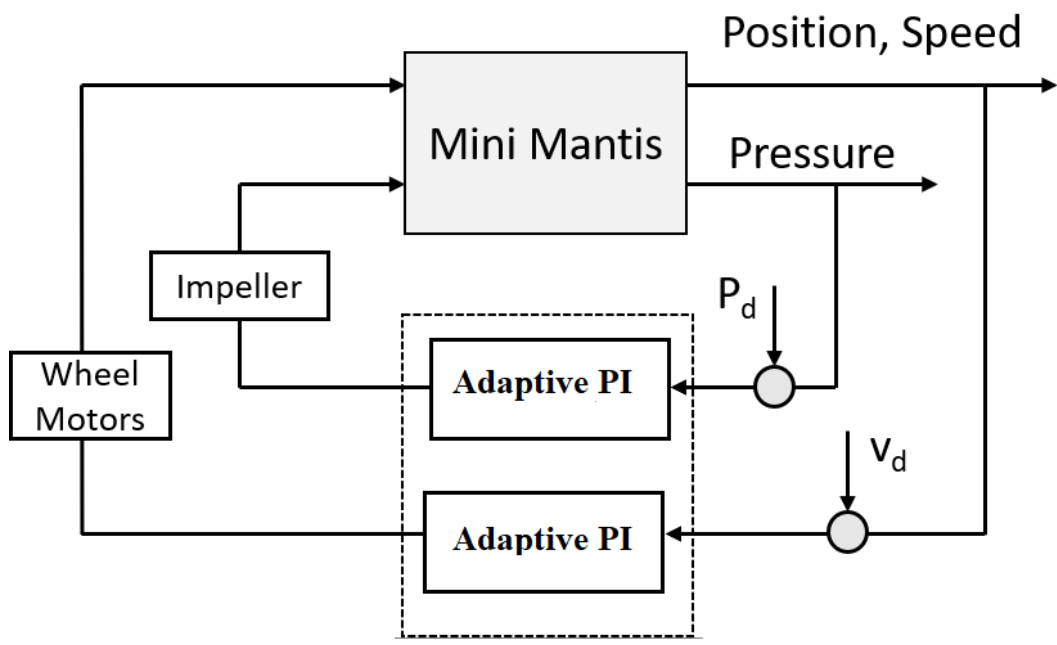

Figure 19. Control Architecture.

A series of experiments involving a single module of the Mantis-mini was conducted to evaluate the adhesion performance of the suction module. The negative pressure is generated by an impeller, which is driven by a DC motor. As the relation between pressure exerted by the impeller and the speed of the DC motor is unknown, multiple experiments are conducted on the impeller. For different type of surfaces (wood, glass, and acrylic), the speed of the motor is varied and the corresponding pressure is measured. Figure 20a shows the variation of the pressure with speed for the $3 \mathrm{~mm}$ central opening of the suction module. Figure 20b shows the variation of the pressure with speed for the $6 \mathrm{~mm}$ central opening of the suction module. From the data plotted in Figure 20, it can be concluded that the speed-pressure relation is approximately linear. Therefore, the pressure can be indirectly controlled by modulating the speed of the motor. 


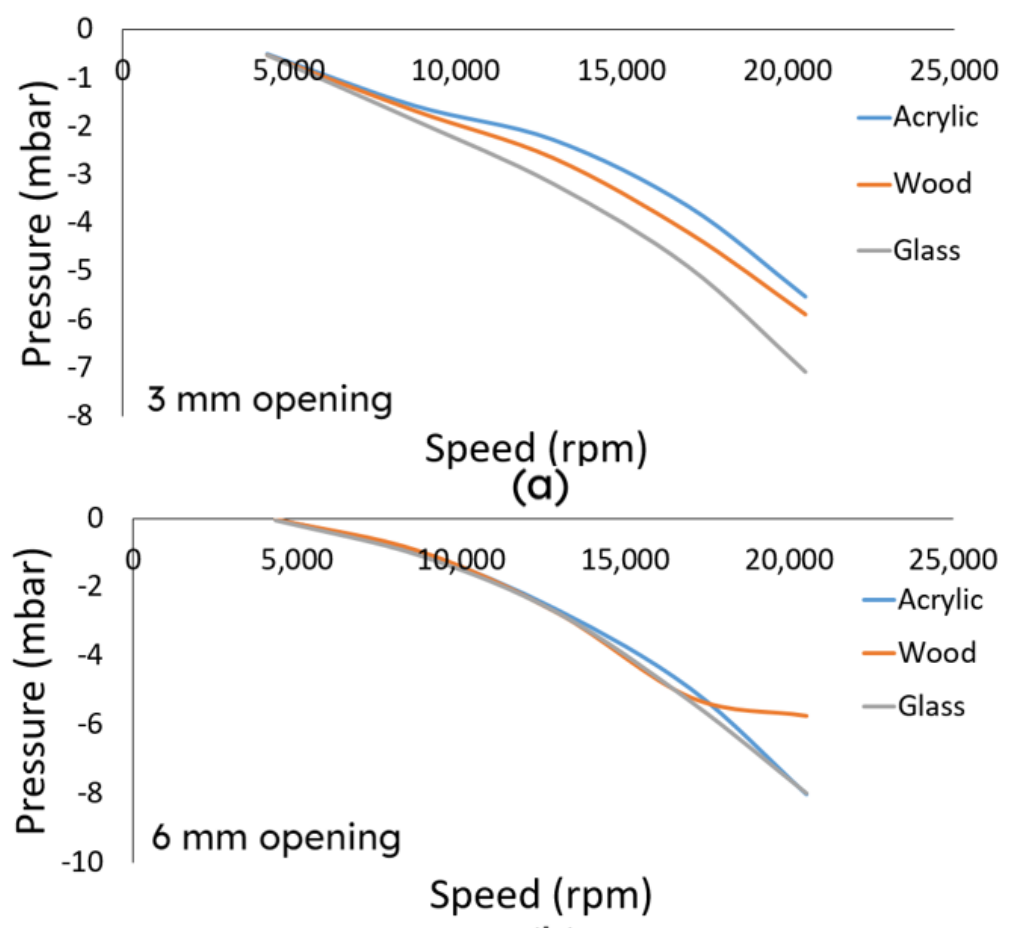

(b)

Figure 20. Variation of pressure with respect to speed on different surfaces by placing the pressure sensor near the central region of suction module with (a) $3 \mathrm{~mm}$ opening and (b) $6 \mathrm{~mm}$ opening.

From the above discussion, it is clear that both the pressure and speed can be controlled through a similar control algorithm. Given a desired speed set-point, a suitably tuned PI controller is capable enough to fulfil the task. However, in the present study the impeller model parameters are unknown and the model may be uncertain. Therefore, an adaptive PI controller [28] is chosen to fulfil the control objective. The DC motor dynamics [29] can be expressed as

$$
\dot{\omega}=\frac{-b}{J} \omega+\frac{K}{J} i+d
$$

where $\omega$ is the motor speed, $i$ the input current, $K$ the torque constant, $J$ represents moment of inertia of the motor, $b$ viscous friction parameter which determines damping, and $d$ a slow varying unknown but bounded disturbance acting on the motor. As the model parameters are unknown and disturbances are common in practical applications, the adaptive PI controller is designed as:

$$
\begin{gathered}
u=-k_{c}(t) \cdot\left(\omega(t)-\omega_{d}\right)-\int_{0}^{t} k_{i}(\tau) \cdot\left(\omega(\tau)-\omega_{d}\right) d \tau \\
k_{c}(t)=c_{1} \cdot\left(\omega(t)-\omega_{d}\right)^{2}+c_{2} \cdot k_{a}(t), k_{i}(t)=c_{3} \cdot k_{a}(t) \\
\dot{k}_{a}(t)=\left(\omega(t)-\omega_{d}\right)^{2}
\end{gathered}
$$

where $\omega_{d}$ is the desired speed of the motor and $c_{i}, i=1,2,3$ are positive scalars. The advantage of the control law (12) is that the control gain does not need tuning and the controller is robust to slow varying disturbances. The control input can be implemented through the PWM technique, and the desired speed can be chosen according to the desired pressure (from Figures 16 and 17).

Generally, the PI gains are tuned by trial and error to achieve a good response. The problem with such approach is that we have to tune the controller parameters (PI gains) $k_{i}$ and $k_{c}$ for every new environment (glass façade). The proposed adaptive controller makes the gains adaptive by making them dependent upon another variable $k_{a}$ which is 
evolving according to the square of the error $\left(\omega(t)-\omega_{d}\right)^{2}$ (As per Equation (12)). If the error increases/decreases, the variable $k_{a}$ increases/ decreases rapidly (proportional to the square of error), ultimately leading to change in $k_{c}$ and $k_{i}$. Hence, the controller adapts automatically depending upon the error and tuning is not necessary.

\subsection{Suction Performance with Controller}

The control algorithm was implemented in the platform and the suction performance was tested. It is found that each module is able to balance itself in a static manner for a range of -0.4 mbar to -0.9 mbar. However, the smooth motion is obtained when the measured pressure is in the range of -0.4 bar. Therefore, the desired pressure commands are set at $(-0.8,-0.4) \mathrm{mbar}$ for the static and the dynamic case. The closed loop performance is plotted in the Figure 21. It is observed that there exists a short delay for the impeller to reach the desired pressures. However, the controller is able to provide a satisfactory regulation both for the static and dynamic cases. The variations in the measured pressure is due to sensor and environmental noise.

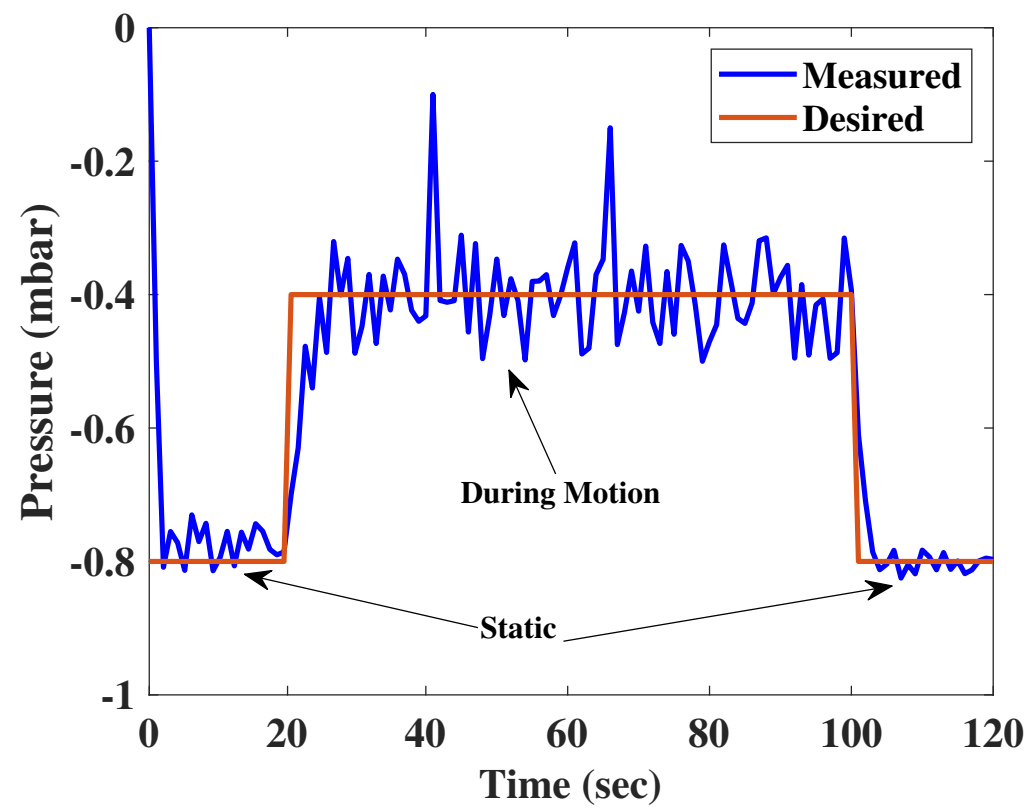

Figure 21. Closed loop performance of the impeller.

\subsection{Locomotion Performance}

We have also conducted experiments to study and analyze the locomotion along different trajectories. These paths include the circular shaped, S-shaped as well as the Z-shaped motion. Similar to the prior experiments, the locomotion trajectories were carried out by the platform and the motor velocity in quadrature pulses per second (qpps) of the various motion trajectories is displayed in Figure 22a-c respectively. From the experiment, we can see that the platform has the ability to move along a curved path based on the differential wheel drive principle. 


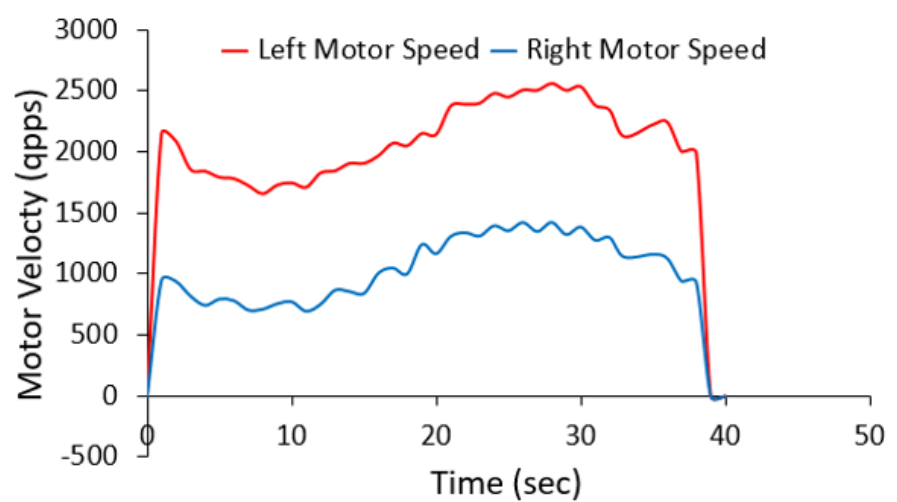

(a)

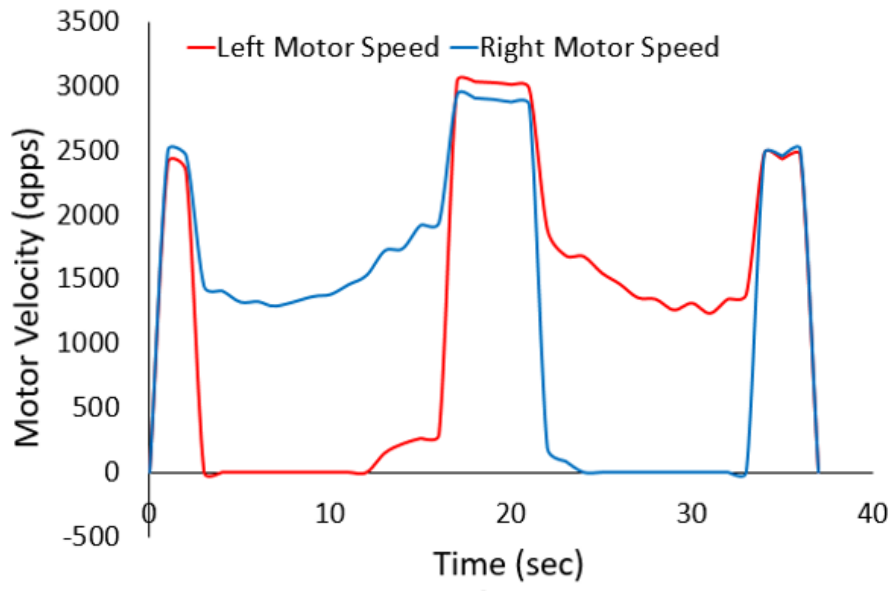

(b)

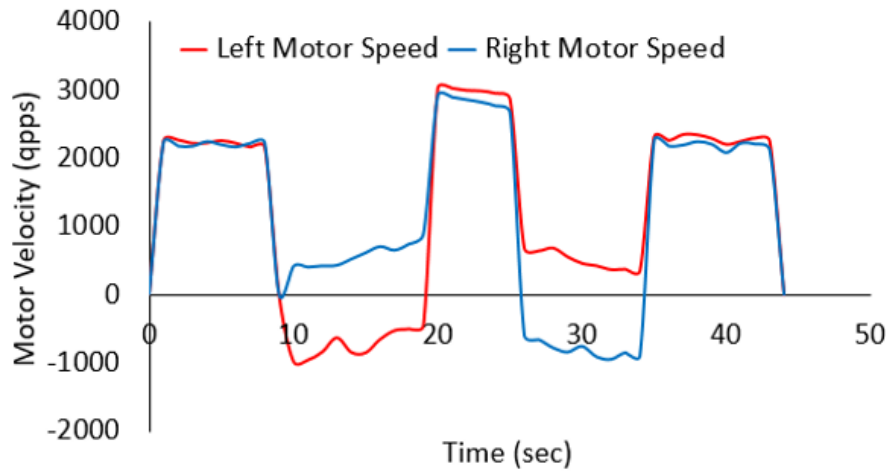

(c)

Figure 22. Locomotion of the Mantis-mini platform along (a) circular, (b) s-shaped (c) z-shaped trajectory.

\section{Conclusions}

A vertical robot was built for cleaning glass façde and transiting the frames. The robot is equipped with suction modules. Each suction module consists of an impeller that generates differential pressure via the central opening of the module and provides adhesion force while navigating on the surface. The linear actuator provides translation movement to each module and as a result each module can be lifted up and perform transition to the next panel. The structural analysis was carried out to verify the structural deformation, frequency behaviour of the platform. Based on the first natural frequency of the platform, the speed of impeller and wheel motors are set to avoid the structural resonance of the system, which is essential for the safety of the system. For this Mantis-mini robot to be static on the glass or to navigate without slipping and falling off, the balance among weight of the robot, dimension, surface type and suction pressure is required. The suction phenomenon 
in the suction chamber was studied analytically and numerically and compared with the experimental results.

CFD simulations showed that the suction pressure magnitude depends on both the impeller speed and the size of base opening. The suction pressure magnitude increases with the impeller speed. The size of the base opening affects the suction pressure as well. Larger opening is more favourable as it leads to a more pronounced axial velocity gradient and higher suction pressure.

Analytical estimation of the suction pressure magnitude was derived and shown to give a fair prediction of the order of magnitude of the suction pressure as well as similar trend with experiments and simulations. As a zero-order modelling tool, this analytical formula could therefore be helpful in estimating the impeller speed to produce the required suction pressure. The latter can be estimated given the weight of the unit, contact area, and friction coefficient. From the simulation, we can also predict the minimum impeller rpm and flow pattern inside the chamber needed to prevent the robot from sliding off at that pressure.

For controlling the adhesion force, we use an adaptive proportional-integral based controller that maintains the minimum amount of force for the robot to remain on the wall. A pressure sensor is attached to suction chamber for monitoring the differential pressure and the error signal is calculated as the difference of the desired impeller speed and the actual rpm. Results show that the implementation of adaptive PI controller helps the platform to follow the trajectory. However, it is noted that the motor velocity along a curved path is not consistent throughout the motion. There are instances where the motor velocity gradually increases or decreases. This is caused by the effect of gravity, when the curved path consist of instances as the platform is heading downwards or upwards. Hence, there will be a need for heading controller using inertial measuring unit as part of the future work.

Author Contributions: Conceptualization, R.P.; Methodology, R.P. and M.M.R.; Analysis, R.P., M.R.E., Z.S.S., T.N. and M.M.R.; Discussion, R.P., M.R.E., Z.S.S., T.N. and M.M.R.; Writing and Revision, R.P., M.R.E., Z.S.S., T.N. and M.M.R. All authors have reviewed and agreed to the published version of the manuscript.

Funding: This research is supported by the National Robotics Programme under its Robotics Enabling Capabilities and Technologies (Funding Agency Project No. 19225 00051), National Robotics Programme under its Robot Domain Specific (Funding Agency Project No. 19222 00058) and administered by the Agency for Science, Technology and Research.

Institutional Review Board Statement: Not applicable.

Informed Consent Statement: Not applicable

Data Availability Statement: No new data were created or analyzed in this study. Data sharing is not applicable to this article.

Conflicts of Interest: The authors declare no conflict of interest.

\section{References}

1. InCeNSE_Incubator. Available online: http:/ /incubation.cense.iisc.ac.in/ (accessed on 23 May 2020).

2. How Dangerous Is High Rise Window Cleaning? 2019. Available online: https://skyswc.com/how-dangerous-is-high-risewindow-cleaning (accessed on 23 May 2020).

3. Kim, T.Y.; Kim, J.H.; Seo, K.C.; Kim, H.M.; Lee, G.U.; Kim, J.W.; Kim, H.S. Design and control of a cleaning unit for a novel wall-climbing robot. In Applied Mechanics and Materials; Trans Tech Publications Ltd.: Schwyz, Switzerland, 2014; Volume 541, pp. 1092-1096.

4. Tomiyama, T.; García, L.R.; Kršlin, A.; Taykaldiranian, G. Systems and conceptual design of a train cab front cleaning robot. Procedia Cirp 2017, 59, 61-66. [CrossRef]

5. Longo, D.; Muscato, G. The Alicia/sup 3/climbing robot: A three-module robot for automatic wall inspection. IEEE Robot. Autom. Mag. 2006, 13, 42-50. [CrossRef]

6. Zhang, H.; Zhang, J.; Zong, G.; Wang, W.; Liu, R. Sky cleaner 3: A real pneumatic climbing robot for glass-wall cleaning. IEEE Robot. Autom. Mag. 2006, 13, 32-41. [CrossRef] 
7. Kim, T.; Seo, K.; Kim, J.; Kim, H.S. Adaptive impedance control of a cleaning unit for a novel wall-climbing mobile robotic platform (ROPE RIDE). In Proceedings of the 2014 IEEE/ASME International Conference on Advanced Intelligent Mechatronics, Besançon, France, 8-11 July 2014; pp. 994-999.

8. Mir-Nasiri, N.; J, H.S.; Ali, M.H. Portable Autonomous Window Cleaning Robot. Procedia Comput. Sci. 2018, 133, 197-204. [CrossRef]

9. Zhang, H.; Zhang, J.; Liu, R.; Zong, G. A novel approach to pneumatic position servo control of a glass wall cleaning robot. In Proceedings of the 2004 IEEE/RSJ International Conference on Intelligent Robots and Systems (IROS) (IEEE Cat. No. 04CH37566), Sendai, Japan, 28 September-2 October 2004; Volume 1, pp. 467-472.

10. Vishaal, R.; Raghavan, P.; Rajesh, R.; Michael, S.; Elara, M.R. Design of Dual Purpose Cleaning Robot. Procedia Comput. Sci. 2018, 133, 518-525. [CrossRef]

11. Hobot Company. 2017. Available online: https://www.hobot.com.tw/Products_list_Window_Robot_298.php (accessed on 23 May 2020).

12. Ecovacs Robotics. 2006. Available online: https://www.ecovacs.com/global/winbot-window-cleaning-robot (accessed on 23 May 2020).

13. Mamibot Manufacturing USA Inc. Available online: https://www.mamibot.com/robot-window-cleaner (accessed on 23 May 2020).

14. Gladwell Company. 2016. Available online: https://www.gladwellclean.com/products/gecko-robot-window-cleaner (accessed on 23 May 2020).

15. Tun, T.T.; Elara, M.R.; Kalimuthu, M.; Vengadesh, A. Glass facade cleaning robot with passive suction cups and self-locking trapezoidal lead screw drive. Autom. Constr. 2018, 96, 180-188. [CrossRef]

16. Menon, C.; Murphy, M.; Sitti, M. Gecko inspired surface climbing robots. In Proceedings of the 2004 IEEE International Conference on Robotics and Biomimetics, Shenyang, China, 22-26 August 2004; pp. 431-436.

17. Anwari, H.; Abdulaziz, N. Design and manufacture of a pneumatic glass climbing robot for cleaning of high-rise buildings. In Proceedings of the 2011 IEEE GCC Conference and Exhibition (GCC), Dubai, United Arab Emirates, 19-22 February 2011; pp. 461-464.

18. Parween, R.; Le, A.V.; Shi, Y.; Elara, M.R. System level modeling and control design of htetrakis-a polyiamond inspired self-reconfigurable floor tiling robot. IEEE Access 2020, 8, 88177-88187. [CrossRef]

19. Parween, R.; Shi, Y.; Parasuraman, K.; Vengadesh, A.; Sivanantham, V.; Ghanta, S.; Mohan, R.E. Modeling and analysis of hHoneycomb-A polyhex inspired reconfigurable tiling robot. Energies 2019, 12, 2517. [CrossRef]

20. Parween, R.; Heredia, M.V.; Rayguru, M.M.; Abdulkader, R.E.; Elara, M.R. Autonomous self-reconfigurable floor cleaning robot. IEEE Access 2020, 8, 114433-114442. [CrossRef]

21. Parween, R.; Prabakaran, V.; Elara, M.R.; Vengadesh, A.; Sivanantham, V. Application of tiling theory for path planning strategy in a polyiamond inspired reconfigurable robot. IEEE Access 2018, 7, 6947-6957. [CrossRef]

22. Le, A.V.; Parween, R.; Kyaw, P.T.; Mohan, R.E.; Minh, T.H.Q.; Borusu, C.S.C.S. Reinforcement Learning-Based Energy-Aware Area Coverage for Reconfigurable hRombo Tiling Robot. IEEE Access 2020, 8, 209750-209761. [CrossRef]

23. Parween, R.; Wen, T.Y.; Elara, M.R. Design and Development of A Vertical Propagation Robot for Inspection of Flat and Curved Surfaces. IEEE Access 2020, 9, 26168-26176. [CrossRef]

24. Hayat, A.A.; Parween, R.; Elara, M.R.; Parsuraman, K.; Kandasamy, P.S. Panthera: Design of a reconfigurable pavement sweeping robot. In Proceedings of the 2019 International Conference on Robotics and Automation (ICRA), Montréal, QC, Canada, 20-24 May 2019; pp. 7346-7352.

25. Nansai, S.; Onodera, K.; Veerajagadheswar, P.; Rajesh Elara, M.; Iwase, M. Design and Experiment of a Novel Façade Cleaning Robot with a Biped Mechanism. Appl. Sci. 2018, 8, 2398. [CrossRef]

26. Vega-Heredia, M and Mohan, R Elara and Wen, Tan Yeh and Siti'Aisyah, J and Vengadesh, A and Ghanta, S and Vinu, S Design and modelling of a modular window cleaning robot. Autom. Constr. 2019, 20, 268-278.

27. Vega-Heredia, M.; Ilyas, M.; Ghanta, S.; Vengadesh, A.; Aisyah, S.; Elara, M.R. Multi-Sensor Orientation Tracking for a Façade-Cleaning Robot. Sensors 2020, 20, 1483. [CrossRef] [PubMed]

28. Fan, J.C.; Kobayashi, T. A simple adaptive pi controller for linear systems with constant disturbances. IEEE Trans. Autom. Control 1998, 43, 733-736. [CrossRef]

29. Kisacanin, B.; Agarwal, G.C. Linear Control Systems: With Solved Problems and MATLAB Examples; Springer Science \& Business Media: Berlin/Heidelberg, Germany, 2012. 